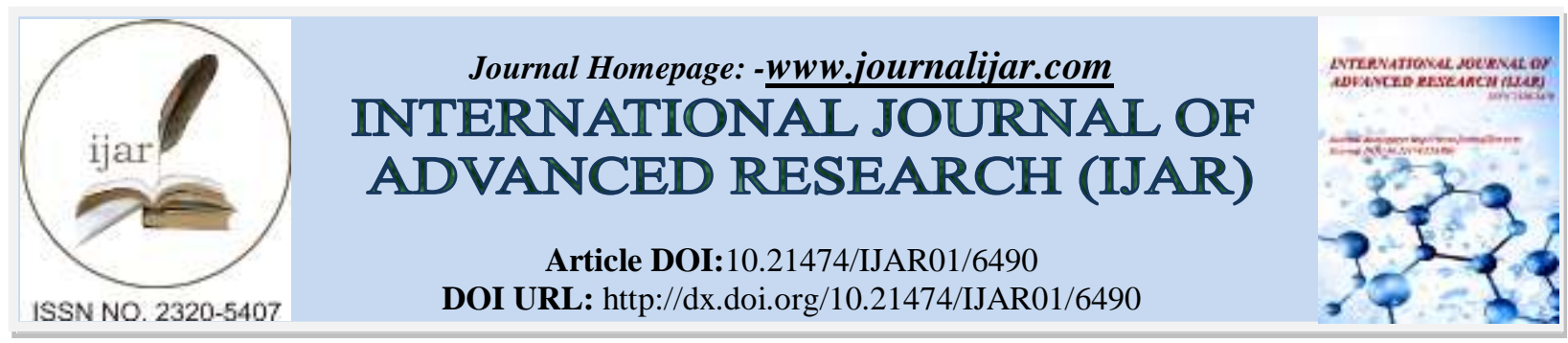

RESEARCH ARTICLE

\title{
LE SYSTÈME DE MANAGEMENT INTÉGRÉ QUALITÉ-SÉCURITÉ-ENVIRONNEMENT APPLIQUÉ À UNE PME.
}

Lagdim Soussi Lalla Hind.

Ecole nationale de commerce et de gestion-Agadir, Université IBNO ZOHR, Maroc.

\section{Manuscript Info}

Manuscript History

Received: 10 December 2017

Final Accepted: 12 January 2018

Published: February 2018

\section{Keywords:-}

Quality management system - Safety management system - Environmental management system - Integrated management system - Integration strategy.

\begin{abstract}
Designing a management system that integrates the three areas of quality, safety and the environment (QSE) is a fundamental challenge for any company concerned with meeting the expectations of all its stakeholders: customers, staff, shareholders but also its environment and society in general.

While the integration of QSE management systems is now gaining increasing interest within the scientific community, empirical research on the integration of management systems at the level of small and medium-sized enterprises is curiously scarce.

However, often faced with the inadequacy of their human and financial resources, SMEs, more than large companies, have every incentive to implement a IMS QSE in order to better structure themselves and be able to perpetuate their future.

This paper studies the integrated management system Quality-SafetyEnvironment (IMS QSE) within the SME to highlight the specificities of this type of company as regards the integration strategy, the organization mode as well as the contributions of the IMS appreciated in relation to the satisfaction of the stakeholders.
\end{abstract}

Copy Right, IJAR, 2018,. All rights reserved.

\section{Introduction:}

Aujourd'hui, face à l'obligation des entreprises de satisfaire les besoins de toutes les parties prenantes pour pouvoir survivre dans un environnement extrêmement concurrentiel et une législation sur la sécurité et l'environnement de plus en plus rigoureuse, le SMI QSE devient un objet académique en pleine expansion.

Concevoir un système de management qui intègre les trois domaines qualité, sécurité, environnement (QSE) constitue un enjeu fondamental pour toute entreprise soucieuse de répondre aux attentes de l'ensemble de ses interlocuteurs : clients, personnel, actionnaires mais aussi son environnement et la société dans son ensemble.

Si l'intégration des systèmes de management QSE suscite désormais un intérêt croissant au sein de la communauté scientifique, les recherches empiriques sur l'intégration des systèmes de management au niveau des petites et moyennes entreprises sont curieusement quasi rares. En effet, les quelques études qui ont été réalisées auprès des PME ont porté essentiellement sur les avantages de l'intégration (enquête réalisée par DOUGLAS et GLEN (2000) sur $28 \mathrm{PME}$ ) ou encore les motivations et barrières à l'intégration des systèmes de management (enquête réalisée par STAMOU (2003) sur 37 PME). 
Force est de remarquer également que la plupart des écrits sur le SMI QSE ne font pas de distinction entre la grande et la petite et moyenne entreprise.

Pourtant, souvent confrontée à l'insuffisance de leurs ressources humaines et financières, la PME, plus que la grande entreprise, a tout intérêt de mettre en œuvre un SMI QSE pour mieux se structurer et pouvoir pérenniser son avenir. Par ailleurs, et en se référant aux auteurs qui revendiquent la spécificité des PME (Torres 1999, Marchesnay 1991) , il parait donc légitime d'apporter une meilleure compréhension de la mise en œuvre du SMI QSE, particulièrement au sein des PME.

La problématique qui s'en suit peut être formulée dans les termes suivants : «Quels sont les spécificités du SMI QSE de la PME ?»

Nous nous intéressons principalement à la stratégie de mise en œuvre, le mode d'organisation ainsi que les apports du SMI QSE quant à la satisfaction des parties prenantes de la PME.

Notre article a donc pour dessein de répondre à cette problématique en présentant dans un premier temps le cadre théorique du SMI QSE, avant d'expliciter notre méthodologie et d'exposer les résultats d'une étude de cas d'une petite et moyenne entreprise marocaine, opérant dans l'industrie métallique.

\section{1- Fondements théoriques du SMI QSE:}

Défini comme une démarche managériale volontaire engagée par la direction de l'entreprise, le système de management intégré (SMI ${ }^{1}$ est un système qui intègre dans une même organisation des processus, la prise en compte d'exigences internes et externes multiples : qualité, sécurité, environnement, exigences légales et réglementaires et d'autres exigences des référentiels propres à l'activité (industrie, services, santé...).

Il n'y a pas d'unanimité entre les différents auteurs sur les composants d'un SMI. Bien que certains auteurs aient suggéré l'intégration d'autres éléments dans le SMI (voir, par exemple, MATIAS et COELHO 2002, RAOUF 2004), la plupart des publications discutent de l'intégration des systèmes de management de la qualité, de l'environnement, de la santé et la sécurité au travail (KARAPETROVIC, 2002) puisque la majorité des organisations industrielles sont tenues de répondre aux exigences réglementaires de l'environnement et de la sécurité, imposées par les gouvernements et les différents groupes d'intérêt. Toutefois, la littérature récente inclut également la responsabilité sociale des entreprises (RSE) comme élément essentiel du SMI en raison de l'accroissement du cercle des parties intéressées.

Selon WILKINSON et DALE (1999), le champ d'un « vrai SMI» devrait être beaucoup plus large que les trois domaines (QSE) et englober tout système pouvant émerger dans le futur (BECKMERHAGEN et al, 2003).

Par ailleurs, plusieurs stratégies d'intégration des systèmes de management ont été proposées par différents auteurs

(KARAPETROVIC et WILLBORN 1998, LABODOVA 2004, WILKINSON et DALE 2001).

Évoquer les stratégies d'intégration des systèmes de management QSE consiste à répondre principalement à la question suivante : «Quels systèmes de management l'entreprise va-t-elle intégrer et dans quel ordre ? »

La plupart des auteurs (SEGHEZZI et SCHWEICKARDT 2001, LISSENDEN 1999) ont suggéré la stratégie d'intégration qui consiste en la mise en œuvre du système de management de la qualité suivi par l'intégration du système de management de l'environnement. D'ailleurs, l'étude empirique de SALOMONE (2008), basée sur une recherche dans des entreprises italiennes, montre comment dans la pratique, une petite majorité des organisations mettent en œuvre le SMQ d'abord, puis par la suite le SME (52\% des entreprises de l'échantillon). Ces résultats sont sensiblement les mêmes que ceux fournis par KARAPETROVIC et al. (2006) à partir d'une étude des entreprises dans la région espagnole de Catalogne, mais dans ce cas le pourcentage de cas a atteint $86 \%$.

Karapetrovic \& Willborn (1998) Ont Proposé quant À eux trois stratégies d'intégration:

1. Mise en œuvre du SMQ en premier suivie par le SME

2. Mise en œuvre du SME en premier, suivie par le SMQ

3. Mise en œuvre du SMQ et SME simultanément

\footnotetext{
${ }^{1}$ Selon Faucher.S (2006), par convention, on parle plutôt de « système de management intégré » ou en abrégé «SMI », mais il serait plus juste de parler de système intégré de management. Il s'agit bien d'intégrer, dans un seul système, toutes les composantes de fonctionnement de l'entreprise : qualité, sécurité, environnement, finance........ et non pas d'avoir un management intégré.
} 
Selon LABODOVA (2004), l'intégration des systèmes de management pourrait se réaliser de deux façons:

1. L'introduction de systèmes individuels (SMQ, SME et SMSS), suivie par leur intégration.

2. Le développement et la mise en œuvre d'un système de management intégré dés le début.

Toutefois, notre lecture de la littérature sur les stratégies d'intégration des systèmes de management nous fait remarquer qu'aucune distinction entre grande entreprise et petite n'a été opérée.

Pourtant, si la petite et moyenne entreprise diffère indéniablement de la grande entreprise en termes de taille, de ressources, de potentialités ..., il est donc légitime à notre sens de penser qu'une stratégie d'intégration lui soit spécifique.

\section{2-Système de management QSE et PME:}

Au niveau mondial, la contribution des PME dans le produit national représente environ $70 \%$, même si cela peut varier considérablement d'un pays à un autre (O'LAOIRE et WELFORD 2014). HILLARY (2000) affirme également que les PME fournissent et créent des emplois, surtout en périodes de récession; elles sont source d'innovation et d'esprit d'entrepreneuriat.

Toutefois, le manque de ressources, de compétences techniques, de temps et d'argent mènent habituellement les PME à l'inaction (JAMES et al. 1998). Les PME sont généralement des entreprises étroitement intégrées au tissu de la collectivité locale, et qui utilisent souvent des procédés et services traditionnels (SMITH 1997). Tandis que les grandes entreprises, disposent du capital pour employer des consultants externes ou peuvent même recruter des experts. Chose qui favorise la recherche d'innovations dans le domaine des systèmes de management (JAMES et al. 1998).

Intégrer les préoccupations qualité-Environnement -sécurité est une nécessité pour toute PME voulant survivre dans un marché mondialisé considérant la satisfaction des parties prenantes comme critère majeur de l'évaluation de la performance. Certes, le SMI QSE présente des bénéfices avérés pour la PME, il faut admettre par contre que sa mise en œuvre se heurte à plusieurs barrières ou difficultés.

\section{1- Bénéfices SMI pour la PME:}

La mise en œuvre des systèmes de management QSE et leur intégration présente pour la PME plusieurs avantages.

L'amélioration de l'efficacité interne et de la qualité du management est le premier bénéfice interne que peut acquérir une petite ou moyenne entreprise. Ceci se fait par exemple, par la réduction de trois départements fonctionnels (Qualité, sécurité, environnement) en un seul (SHILLITO 1995) et l'harmonisation des structures organisationnelles qui contiennent des éléments similaires (WINDER 2000).

De nombreux auteurs (par exemple RENZI et CAPPELLI 2000, GRIFFITH 2000) reconnaissent également la diminution de la documentation à travers la création de documents communs qui peuvent être utilisés plus facilement par plusieurs opérationnels.

Les PME peuvent également obtenir un ensemble d'avantages financiers. Les économies de coûts qui découleront de la diminution de la fréquence des audits, sont reconnues dans la littérature (voir par exemple SHILLITO 1995, WINDER 2000, MATIAS et COELHO 2002), bien que SHILLITO (1995) souligne que les audits ne sont pas réduits à un seul, car il faudra élargir le programme d'audit financier interne afin d'assurer l'efficacité des procédures intégrées.

En somme, STAMOU (2003) a catégorisé l'ensemble des bénéfices cités dans la littérature, qu'une PME peut tirer de l'intégration des systèmes de management, en bénéfices internes et bénéfices externes comme le montre le tableau 1.

Tableau 1:-Bénéfices du SMI QSE implanté par la PME(traduit et modifié de STAMOU (2003) par nous-mêmes Bénéfices internes

\begin{tabular}{|c|c|}
\hline 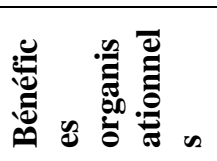 & $\begin{array}{l}\checkmark \text { Amélioration de la qualité de gestion en réduisant trois départements fonctionnels en un } \\
\text { seul } \\
\checkmark \text { Augmentation de l'efficacité opérationnelle à travers l'harmonisation des structures } \\
\text { organisationnelles des différents systèmes individuels et le partage d'information }\end{array}$ \\
\hline
\end{tabular}




\begin{tabular}{|l|ll|}
\hline & $\checkmark$ & Elimination des dédoublements des procédures des systèmes \\
& $\checkmark$ & Rationalisation de la documentation et de la communication \\
\hline & $\checkmark$ & Économies de coûts grâce à la réduction de la fréquence des audits \\
& $\checkmark$ & Réduction des coûts de certification externe par rapport aux audits de certification uniques \\
& $\checkmark$ & Augmentation des marges bénéficiaires \\
\hline & $\checkmark$ & Augmentation de la motivation, de la sensibilisation et des qualifications des employés \\
\hline & $\checkmark$ & Création d'une meilleure image de l'entreprise chez les employés \\
\hline & & \\
\hline Bénéfices externes & & \\
\hline & & \\
\hline
\end{tabular}

Selon certains auteurs, l'implantation d'un système de management, et par conséquent du SMI, est non seulement techniquement appropriée pour les petites et moyennes entreprises, mais elle peut être considérablement plus facile pour elles que les grandes entreprises.

WELLS et GALBRAITH (1999) utilise l'ISO 14001 pour illustrer cet argument, en affirmant que les petites et moyennes entreprises peuvent l'adopter plus facilement en raison de leurs petites tailles, qui se traduisent par moins d'impacts environnementaux significatifs, moins de bureaucratie au sein de l'entreprise, et une formation et communication interne plus faciles.

Néanmoins, et comme il se produit dans tout autre projet ayant de fortes implications managériales, le processus d'intégration est également accompagné de barrières ou difficultés qui devraient être prises en considération afin d'éviter l'échec.

\section{2- Difficultés et barrières du SMI pour la PME:}

Plusieurs barrières à la mise en œuvre du SMI QSE, d'ordre interne (ressources, attitudes/ perceptions, implantation) et externe (soutien et direction, économie, certification et vérification) peuvent se dresser devant les entreprises, grandes ou petites, comme le montre le tableau 2.

\begin{tabular}{|l|l|}
\hline \multicolumn{2}{|l|}{$\begin{array}{l}\text { Tableau 2:-Difficultés de l'intégration des systèmes individuels de management(traduit et modifié d'ASIF et al } \\
\text { (2008) par nous-mêmes) }\end{array}$} \\
\hline Difficultés de l'intégration & Sources de la littérature \\
\hline Diversité des constituants des systèmes de management \\
\hline $\begin{array}{l}\text { Champs d'application différents des systèmes individuels de } \\
\text { management }\end{array}$ & $\begin{array}{l}\text { WILKINSON et DALE (2001); ZENG et al } \\
(2007)\end{array}$ \\
\hline Structures différentes des systèmes individuels de management & $\begin{array}{l}\text { KARAPETROVIC (2002) ; SEGHEZZI, } \\
(2001)\end{array}$ \\
\hline Méthodologies d'audit inadéquates & KARAPETROVIC (2002) \\
\hline Indisponibilité d'un SMI formel & LABODOVA (2004) \\
\hline Absence d'un dénominateur commun & KARAPETROVIC (2002) \\
\hline Incompréhension du concept d'intégration & WILKINSON et DALE (2001) \\
\hline Résistance des employés & $\begin{array}{l}\text { MATIAS et COELHO (2002); ZUTSHI et } \\
\text { SOHAL (2005) }\end{array}$ \\
\hline Rejet des employés du nouveau système &
\end{tabular}




\begin{tabular}{|l|l|}
\hline $\begin{array}{l}\text { Résistance des employés due à une perte de leurs fonctions } \\
\text { individuelles }\end{array}$ & KARAPETROVIC (2002) \\
\hline Manque de ressources & $\begin{array}{l}\text { ZENG et al. (2007) ; ZUTSHI et SOHAL } \\
(2005)\end{array}$ \\
\hline Expertise insuffisante & ZUTSHI et SOHAL (2005) \\
\hline Insuffisance des ressources & CROWE (1992) \\
\hline Difficultés postérieures à la mise en ouvre & MATIAS et COELHO (2002) \\
\hline Réduction de la flexibilité après l'intégration & $\begin{array}{l}\text { JORGENSEN et al. (2005); WILKINSON et } \\
\text { DALE (1999), ZENG et al. (2007) }\end{array}$ \\
\hline Augmentation de la bureaucratie & \\
\hline Aspects culturels & \\
\hline Nécessité d'une transformation culturelle &
\end{tabular}

Par ailleurs, si la plupart de ces difficultés concernent aussi bien la grande entreprise que la petite et moyenne entreprise, certaines sont spécifiques à la grande entreprise comme la complexité de l'entreprise (sites unique / multiple; nationales / multinationales), tandis que d'autres concernent surtout la PME comme le manque de ressources financières et d'expertise.

En effet, beaucoup de petites et moyennes entreprises (PME) ont des ressources insuffisantes pour mettre en œuvre un SMI (ZUTSHI et SOHAL 2005). Chose qui constitue un obstacle important dans l'intégration des systèmes individuels de management et leur mise en œuvre surtout quand des coûts supplémentaires sont engagés pour obtenir la certification par une tierce partie.

Et bien qu'une aide de source externe attribuée au SMI puisse faciliter le processus de sa mise en œuvre, l'internalisation et la maintenance du système revient aux principaux usagers, qui dans le cas des PME ne peuvent pas avoir le niveau d'expertise requis (ASIF et al ,2008).

HILLARY (1999) soutient que les PME peuvent trouver de nombreux problèmes, comme les coûts imprévus et les compétences du personnel, l'incapacité d'intégrer les systèmes en douceur ou encore le manque de bénéfices au niveau du marché.

\section{3- Méthodologie de recherche:}

Pour comprendre le déploiement du SMI QSE au sein de la PME, il a été nécessaire, d'adopter une approche qualitative. Nous avons ainsi effectué une étude de cas d'une entreprise faisant partie du secteur de l'industrie métallique et mécanique et du sous secteur «Travail des métaux ». Son activité consiste en la fabrication de menuiseries et fermetures métalliques. Son effectif ne dépassant pas habituellement une trentaine de personnes, elle est incontestablement comptée parmi les petites et moyennes entreprises (PME).

L'étude de cas en profondeur de cette PME présente ainsi, à notre sens, plusieurs avantages :

-en multipliant les entretiens avec plusieurs intervenants des systèmes de management QSE, cette démarche offre la possibilité de diversifier les visions de l'intégration des systèmes de management QSE et de ne pas se contenter de la perception d'un seul acteur. Ceci permet d'éviter le risque de subjectivité d'un acteur unique ;

-elle permet d'observer directement le fonctionnement de l'intégration des systèmes de management QSE à différents niveaux hiérarchiques ;

-elle permet de recueillir une quantité plus importante d'informations, avec le plus possible de descriptions et de détails sur le SMI QSE que nous voulons étudier.

Ainsi, nous avons recueilli nos données primaires directement sur le terrain comme suit :

- par entretiens individuels : nous avons préféré que ces entretiens soient semi-directifs pour éviter que le discours des interviewés ne s'éloigne du cœur de notre recherche. Nous avons ainsi effectué des entretiens avec le directeur général, avec la responsable QSE et avec quatre pilotes de processus.

- par observation non participante : nous avons passé quelques jours au sein de l'entreprise étudiée. Chose qui nous a permis d'observer les pratiques QSE (Communication sur les aspects QSE, affichages en matière de sécurité, de qualité et d'environnement, pratiques QSE sur les sites de production comme par exemple la fréquence des 
contrôle qualité, le port des équipements de protection individuelle par les salariés au niveau de la sécurité ou encore le rangement des déchets au niveau de l'environnement).

- l'administration d'un questionnaire auprès d'un échantillon de collaborateurs (14 collaborateurs) selon le mode du «face à face », pour évaluer l'impact du SMI QSE sur la satisfaction du personnel, comprenant des questions sur :

1. Le rendement des employés (perception des changements induits par l'intégration, conditions du travail)

2. Le développement des employés (champs des responsabilités, compétences professionnelles),

3. Le climat du travail (satisfaction du personnel, motivation du personnel).

Les données secondaires ont pareillement été d'une grande utilité pour notre recherche. Nous avons ainsi pu consulter :

- la documentation externe : Préalablement à notre étude de cas, nous avons examiné le site internet de la PME étudiée, et notamment les rapports annuels et les rubriques concernant les aspects QSE. Nous avons fait aussi une recherche plus générale sur le Web pour mettre au jour d'éventuels documents externes concernant l'entreprise (présentations lors de journées, articles de presse, apparition de l'entreprise dans certaines études relatives aux domaines QSE, ou dans certains classements comme le prix national de sécurité ou de qualité, etc.). Une partie de ces documents nous a servi par la suite dans notre description et notre analyse des cas.

- la documentation interne de l'entreprise (manuel QSE, cartographie des processus, procédures, modes opératoires et instructions de travail, comptes rendus de revues de direction, tableaux de bord, organigrammes, etc.).

\section{4- Résultats de l'étude empirique:-}

\section{1- Stratégie d'intégration des systèmes de management QSE au sein de la PME étudiée:-}

La stratégie adoptée par l'entreprise pour l'intégration de ses systèmes de management QSE est une stratégie progressive qui consiste en la mise en œuvre d'un système de management de la qualité, suivie par la mise en œuvre et l'intégration des deux autres systèmes de management de l'environnement et de la santé et sécurité au travail. En effet, la première démarche à avoir été initiée par l'entreprise est la démarche qualité. Certes, les préoccupations de l'entreprises quant aux aspects sécurité et environnement étaient également présentes dés la création de l'entreprise, mais leur formalisation ne se fera qu'ultérieurement.

En effet, ce n'est qu'au début de l'année 2000 qu'un processus de certification ISO 9001 V2000 a été engagé formellement par l'entité bien que la recherche de la qualité (pour les produits et, plus globalement, dans la démarche générale de gestion) ait été considérée pour les propriétaires comme l'un des atouts décisif du succès.

Toutefois, la démarche de certification qualité financée préalablement par les propres moyens de l'entreprise, fut interrompue en 2002 à cause du manque des moyens financiers.

Elle est reprise en 2003 dans le cadre du programme MEDA EUROPAID ${ }^{2}$, pour être menée à son terme au début de 2005. L'entreprise a bénéficié grâce à ce programme d'un appui financier et d'une expertise. Par la suite, l'association de parachimie, dans le cadre du Programme d'Appui aux Entreprises (PAE), avait invité les entreprises à faire des «Diagnostics sécurité », tout en mettant à leur disposition une expertise marocaine et étrangère. L'entreprise a répondu à l'appel en réalisant un diagnostic sécurité, qui a permis, outre l'identification et l'évaluation des différents risques de l'entreprise, la proposition d'un plan d'action, préparant ainsi l'entreprise à une nouvelle certification du système de management de la sécurité et du système de management de l'environnement

Finalement, la certification qualité a été obtenue en 2005, suivie par la certification sécurité et environnement en 2008, conformément aux normes Qualité NM ISO 9001-2008, Sécurité NM 00.5.801-2009 et Environnement NM ISO 14001-2006.

D'une manière globale, notre analyse de la stratégie progressive de la mise en œuvre des systèmes de management QSE adoptée par cette PME s'explique principalement par les facteurs suivants :

\footnotetext{
${ }^{2}$ Programme du ministère marocain de commerce et de l'industrie, et de l'Union Européenne pour mettre à niveau les entreprises marocaines. MEDA devait choisir 200 entreprises marocaines pour les accompagner dans la mise en place de la démarche qualité et 50 entreprises pour l'accompagner dans la certification qualité.
} 
- Le manque de moyens financiers : Le coût de la mise en œuvre des trois systèmes de management QSE est énormément élevé et dépasse de loin les moyens financiers des PME marocaines.Par rapport à la grande entreprise, les PME ont toujours éprouvé plus de difficultés à se financer dans la mesure où elles ne bénéficient pas des mêmes sources de financement.Comme le souligne Brio \& Junquera (2002), les PME manquent généralement de ressources humaines et financières pour faire face aux nouvelles pressions ((p. ex. les préoccupations des parties prenantes sur les impacts sur l'environnement ou les nouvelles règlementations sur la santé et de sécurité).

Ainsi, l'entreprise objet de notre étude de cas devait à chaque fois attendre une aide financière externe pour mettre en œuvre l'un des systèmes de management QSE et l'intégrer par la suite avec les autres.

- Le manque d'expertise : Le besoin d'une expertise dans la mise en place des systèmes de management se fait plus sentir au sein des PME que la grande entreprise. En effet, en se référant aux études effectuées par KALIKA (1987) à partir de critères comme la standardisation des procédés, la formalisation des procédures ou encore la séparation fonctionnelle, plus l'entreprise est petite, moins il y a de formalisation. Ainsi, mettre en place un système de management nécessite l'adoption de l'approche processus, la création d'un manuel de management, l'élaboration des procédures et instructions de travail. Bref, il s'agit pour une PME de formaliser tout ce qui se pratiquait en matière de gestion et d'organisation d'une manière informelle. Chose qui demande incontestablement une expertise externe.

\section{2- Organisation des fonctions QSE:}

La petite taille de l'entreprise étudiée fait que l'organisation des fonctions QSE est extrêmement simple. En effet, on retrouve au:

- Niveau stratégique: c'est le directeur générale lui-même qui veille sur le système de management intégré QSE. Chose qui prouve son engagement au développement et à l'amélioration continue de l'efficacité du SM QSE

- Le niveau fonctionnel : nous retrouvous la représentante de la direction (qui est d'ailleurs l'une des actionnaires de l'entreprise). Elle assure, en concertation avec la direction, la mise en œuvre de la politique QSE. Elle joue le rôle de « coordinateur » du système de management et participe aux différentes décisions s'y rapportant. Les fonctions de la représentante QSE de direction sont variées. Elles consistent, entre autres, à :

- Préconiser, mettre en place et suivre les procédures sécurité et environnement

- Assurer le suivi du système qualité et élaborer un schéma directeur permettant de prendre en compte les objectifs de la Direction Générale, les contraintes de l'entreprise et les demandes des clients

- Effectuer des audits internes en matière d'application des process et de la réglementation

- Prendre en charge la veille réglementaire et technologique

- Participer à l'analyse des défaillances

- Faire respecter les exigences légales qui s'appliquent au domaine d'activité

- Sensibiliser les membres de l'entreprise aux enjeux liés à la sécurité, la qualité et l'environnement

- Le niveau opérationnel : ce sont les pilotes des processus qui assurent l'amélioration continue des processus. En effet, les processus ont besoin d'évoluer et il faudra nécessairement faire évoluer les ressources affectées par ces modifications et donc mettre en œuvre les moyens nécessaires associés. Aussi, les pilotes de processus revoient continuellement les indicateurs inhérents à chaque processus, pour ainsi les améliorer, les enrichir, ou dans certains cas, les modifier. Ils ont également, entre autres, la tâche de sensibiliser les acteurs des processus sur le rôle que ces derniers ont à jouer au sein du processus et veillent à ce que les informations concernant le fonctionnement des processus remontent à la direction.

D'une manière générale, nous avons constaté que le nombre réduit des employés ainsi que la proximité de la direction permet de suivre de prés les pratiques QSE, de communiquer continuellement sur les engagements de la direction et de procéder rapidement aux améliorations QSE.

L'information, en sa majorité informelle, circule aisément et rapidement dans l'entreprise. N'importe quel dysfonctionnement au niveau des aspects QSE, ou même au niveau de la production, est immédiatement communiqué au comité de direction (le directeur et la représentante de la direction). Les mesures nécessaires sont alors prises pour y remédier. La communication en interne est fluide et très dynamique du fait de la petite taille de l'entreprise, impliquant ainsi une sensibilisation quotidienne sur les aspects QSE. 
La PME se caractérise bel et bien par un management QSE de proximité qui repose sur des interactions étroites et directes entre le personnel et qui impactent positivement la communication et la gestion de l'information sur les aspects QSE.

\section{3- Apports du SMI QSE à la PME étudiée:}

Nous avons apprécié les bénéfices du SMI QSE pour la PME objet de notre étude, par rapport à la satisfaction de ses parties prenantes (clients, personnel, groupes externes et actionnaires). Il en ressort ce qui suit dans le tableau 3 :

\begin{tabular}{|c|c|}
\hline $\begin{array}{l}\text { Partie } \\
\text { prenantes }\end{array}$ & Bénéfices du SMI QSE \\
\hline Clients & $\begin{array}{l}\text { - Produits de haute qualité } \\
\text { - Taux de satisfaction généralement supérieur à } 9 \text { sur } 10\end{array}$ \\
\hline Personnel & $\begin{array}{l}\text { - Meilleure rendement des employés : } \\
\quad \text { - Organisation du travail devenue facile } \\
\text { - Pas de complexité du système documentaire } \\
\text { - Augmentation des compétences professionnelles suite à l'élargissement à des champs des } \\
\text { responsabilités (Deux ou trois domaines de responsabilité au lieu d'un seul) } \\
\text { - Meilleure satisfaction des salariés } \\
\text { - Meilleure motivation au travail } \\
\text { - Meilleure Sécurité et santé des employés au travail : atteinte de l'objectif du zéro accident }\end{array}$ \\
\hline $\begin{array}{l}\text { Groupes } \\
\text { externes }\end{array}$ & $\begin{array}{l}\text { - Protection des ressources naturelles : Installations disposant d'un circuit interne permettant la } \\
\text { circulation de l'eau, sa filtration et sa réutilisation. } \\
\text { - L'économie d'énergie : Machines économes permettant de minimiser la consommation } \\
\text { d'énergie et d'éviter les pertes et les gaspillages } \\
\text { - Maîtrise de la pollution dégagée : } \\
\text { - Déchets recyclés ou donnés à d'autres entreprises } \\
\text { - Aucune réclamation de la part des riverains et de l'administration }\end{array}$ \\
\hline Actionnaires & $\begin{array}{l}\text { - Réduction des coûts : } \\
\text { - La régression des primes d'assurance } \\
\text { - } \quad \text { La réduction des coûts de l'énergie } \\
\text { - } \quad \text { Réduction des coûts de la non-qualité } \\
\text { - L'amélioration de l'image de l'entreprise : } \\
\text { - Prix national de la Qualité, catégorie PMI, Edition } 2006 \\
\text { - Prix national de la Sécurité au Travail, catégorie PMI, Edition } 2006 \\
\text { - L'entreprise est donnée comme exemple ou modèle dans différentes manifestations } \\
\quad \text { télévisées ou écrites }\end{array}$ \\
\hline
\end{tabular}

Par ailleurs, certains auteurs comme GIBSON (2000, p. 251) soulignent que le concept de partie prenante entretient une certaine confusion entre les individus et les groupes, voire une redondance dans la mesure où un même individu peut fort bien appartenir à plusieurs groupes : il peut être, à la fois, actionnaire, employé, membre de la communauté et consommateur.

Notre recherche montre que le SMI QSE permet de satisfaire à la fois les différents besoins des groupes de parties prenantes, quelque soit l'appartenance de l'individu, et même si ce dernier peut faire partie de plusieurs groupes de parties prenantes.

Nous avons pu remarquer également que le SMI QSE a structuré la PME étudiée de façon à lui permettre d'améliorer ses relations avec les parties prenantes et de les intégrer dans la prise de décision notamment au niveau des investissements et projets futurs de l'entreprise qui tiennent compte des exigences QSE.

Enfin, si l'entreprise étudiée a atteint un niveau d'excellence, c'est grâce aux engagements des dirigeants : leurs convictions et leurs motivations se sont imprégnées de la culture de l'amélioration continue. L'ambition pour une performance multidimensionnelle, permettant de satisfaire toutes les parties prenantes ne fait que croitre. Les projets futurs de l'entreprise comprennent d'autres certifications ainsi que la promotion de la responsabilité sociale. 


\section{Conclusion:-}

Plusieurs enseignements peuvent être tirés de cette recherche. En premier lieu, elle confirme que la petite taille de l'entreprise est à double tranchant : si elle peut poser obstacle en matière des ressources financières et d'expertise, elle constitue en revanche un atout en termes de communication et d'information sur les systèmes de management QSE.

Aussi, grâce au SMI QSE, nous retrouvons une mutualisation des domaines QSE et une meilleure cohérence dans la documentation de l'entreprise. On ne raisonne plus séparément pour chaque domaine, mais globalement. Autrement dit, les trois aspects QSE sont systématiquement traités au niveau de chaque nouvelle procédure, chaque nouvelle instruction de travail ou encore chaque mode opératoire. Il s'agit en fait d'une même démarche avec les mêmes acteurs : le personnel, les clients, les voisins, les groupes externes.

L'implantation du SMI QSE au sein de la PME peut certes faire face à certains obstacles propres à la petite et moyenne entreprise, les bénéfices de ce système sont en revanche considérables et permettent à toute PME de se différencier et de rivaliser la grande entreprise au niveau de la performance devenue aujourd'hui en forte corrélation avec la satisfaction des parties prenantes.

Le SMI QSE constitue pour la PME, sans le moindre doute, un levier de croissance. Plus que des valeurs, c'est un outil pour l'aiguiller vers l'amélioration continue et lui paver la voie au développement durable.

\section{Références bibliographiques:-}

1. ASIF M., DEBRUIJN E-J., FISSCHER O-A-M. et STEENHUIS H-J . (2008). Achieving sustainability three dimensionally. Papier présenté à la $4^{\text {ème }}$ conférence internationale du Management of Innovation and Technology.Bangkok.

2. BECKMERHAGEN I-A., Berg H-P., KARAPETROVIC S-V. et WILLBORN W-O. (2003). Integration of management systems: focus on safety in the nuclear industry. International Journal of Quality and Reliability Management. Vol. 20 No. 2, pp. 210-228.

3. BRIO J.A. et JUNQUERA B. (2002). A review of the literature on environmental innovation management in SME's: implications for public policies. Technovation. Article in Press.

4. CROWE T-J. . (1992) . Integration is not synonymous with flexibility. International Journal of Operations and Production Management, 12(10), pp. 26 -33.

5. DOUGLAS A. , GLEN D. (2000). Integrated management systems in small and medium enterprises », Total Quality Management, Vol. 11 Nos 4-6, pp. 686-90.

6. FAUCHER S. (2006), Système intégré de management: qualité, sécurité, environnement, AFNOR.

7. GIBSON K. (2000 ), The Moral Basis of Stakeholder Theory, Journal of Business Ethics, vol. 26, $\mathrm{n}^{\circ}$ 3, pp. 245-257.

8. GRIFFITH A. (2000), Integrated management systems:a single management system solution for project control? . Engineering, Construction and Architectural Management, Vol. 7, no 3, pp. 232-240.

9. HILLARY R. (1997). Environmental Management Systems and Cleaner Production. Chapter 33, Wiley \& Sons, London.

10. HILLARY R. (2000). ISO 14001: Case Studies and Practical Experiences. Chapter 5. Greenleaf. Sheffield.

11. JAMES P., HOLT D., HOMEWOOD S., et VINEY H. (1998), Sustainable business strategies for the SME sector . In Proceedings of International Sustainable Development Research Conference, pp. 142-147.

12. JORGENSEN T-H., REMMEN A., Mellado M-D. (2005), Integrated management systems-three different levels of integration. Journal of Cleaner Production, 14(08), pp. 713-722.

13. KALIKA M..(1987), Structures d'entreprises. Economica.

14. KARAPETROVIC S.,WILLBORN W. (1998). Integration of quality and environmental management systems . The TQM Magazine, 10(3), pp. 204-213.

15. KARAPETROVIC, S. et WILLBORN, W. (1998a). The system's view for clarification of quality Vocabulary . International Journal of Quality \& Reliability Management, Vol. 15 No. 1, pp. 99-120.

16. KARAPETROVIC S. (2002). Strategies for the integration of management systems and standards .The TQM Magazine, 14, pp. 61-67.

17. KARAPETROVIC S., CASADESU S-M. et HERAS J. (2006). Dynamics and Integration of Standardised Management Systems: An Empirical Study. Documenta Universitaria, Girona. 
18. LABODOVA A. (2004). Implementing integrated management systems using a risk analysis based approach. Journal of Cleaner Production, 12(06), pp. 571-580.

19. LISSENDEN J. (1999) . ISO 9000 eases ISO 14001 registration . Quality Digest, Vol. 19 No. 5, pp. 41-44.

20. MARCHESNAY M.. (1991). La PME: une gestion spécifique . Economie rurale, 206(1), pp.11-17.

21. MATIAS J.C.D.O. et COELHO D.A. (2002). The integration of the standards systems of quality management, environmental management and occupational health and safety management. International Journal of Production Research, Vol. 40, part 15, pp. 3857-3866.

22. O'LAOIRE D. et WELFORD R. . (2014). The EMS in the SME. In: Welford, R. (2014), Corporate Environmental Management 1: Systems and Strategies, Chapter 11, Earthscan, London.

23. RAOUF A. (2004). Productivity enhancement using safety and maintenance integration: An overview. Kybernetes, 33(07), pp. 1116-1126.

24. RENZI M.F. et CAPPELLI L. .(2000). Integration between ISO 9000 and ISO 14000: opportunities and limits. Total Quality Management, Vol. 11, no. 4-6, pp. 849-856.

25. SALOMONE R. . (2008) . Integrated management systems: experiences in Italian organizations . Journal of Cleaner Production, Vol. 16 No. 16, pp. 1786-806.

26. SEGHEZZI H. D., et SCHWEICKARDT S. (2001). Integration of quality management into business management: an IAQ project report. The Best on Quality, 12, pp.3-50.

27. SHILLITO D.E. (1995). Grand Unification Theory' or Should Safety, Health, Environment and Quality be Managed Together or Separately? .Institution of Chemical Engineers, Vol. 73, part B, pp. 195-202.

28. SMITH M. .(1997). Stimulating environmental action in small to medium-sized enterprises. Environmental Management Systems and Cleaner Production, Wiley: Chic ester.

29. STAMOU T. (2003). Integrated Management Systems in Small Medium-Sized Enterprises: Theory and Practice. Thèse (M.Sc.). Norwich. Angleterre, University of East Anglia

30. TORRES O. (1999). «Les PME». Dominos.

31. WELLS R.P. et GALBRAITH D. (1999). Proyecto Guadalajara: Promoting Sustainable Development through the Adoption of ISO 14001 by Small and Medium-Sized Enterprises. Greener Management International, Issue 28, pp. 90-102.

32. WILKINSON G., DALE B-G. (1999). Integrated management systems: an examination of the concept and theory. The TQM Magazine, 11(2), pp. 95-104.

33. WILKINSON G. et DALE B-G. (2001). Integrated management system: a model based on total quality approach. Managing Service Quality, Vol. 11 No. 5, pp. 318-330.

34. WINDER C. (2000). Integrating OHS, Environmental and Quality Management Standards. Quality Assurance: Good Practice Regulation and Law, Vol. 8, part 2, pp.105-135.

35. ZENG S-X., SHI J-J., LOU G-X. (2007). A synergetic model for implementing an integrated management system: an empirical study in China., Journal of Cleaner Production, 15(18), pp. 1760-1767.

36. ZUTSHI A., SOHAL A-S. (2005). Integrated management system: The experiences of three Australian organizations. Journal of Manufacturing Technology Management, 16(02), pp.211-232. 